\title{
An Accessible User Interface Concept for Non-Verbal and Spatial Aspects of Business Meetings for Blind and Visually Impaired People
}

\author{
Reinhard Koutny* \\ reinhard.koutny@jku.at \\ Johannes Kepler University Linz \\ Linz, Austria
}

\author{
Klaus Miesenberger \\ klaus.miesenberger@jku.at \\ Johannes Kepler University Linz \\ Linz, Austria
}

\begin{abstract}
Business meetings are an integral part of work life. While the modus operandi has changed over the course of the past years and decades, shifting from meetings with analog tools like flipcharts to digital aids like projectors or large touch displays mounted to walls, some aspects have stayed the same: Information does not only get exchanged verbally. Visual information like flipcharts but also non-verbal communication and spatial information are deemed essential information. This information is basically inaccessibly to blind and visually impaired people, which renders business meetings inaccessible to them as well. Consequentially, BVIPs have a tremendous disadvantage in their professional life. While research has been concerned with the mitigation of single aspects of the whole problem, especially with tracking all types of information, no comprehensive and user-friendly user interface approach has been developed. This paper proposes an accessible user interface approach facilitating affordable off-the-shelf hardware, which allows access to visual, non-verbal and spatial aspects of business meetings in a user-friendly manner and presents a fully functional prototype called MAPVI brainstorming tool.
\end{abstract}

\section{CCS CONCEPTS}

- Human-centered computing $\rightarrow$ Interaction paradigms; Accessibility theory, concepts and paradigms.

\section{KEYWORDS}

business meetings, brainstorming meetings, blind and visually impaired people, accessibility

\section{ACM Reference Format:}

Reinhard Koutny and Klaus Miesenberger. 2021. An Accessible User Interface Concept for Non-Verbal and Spatial Aspects of Business Meetings for Blind and Visually Impaired People. In Mensch und Computer 2021 (MuC '21), September 5-8, 2021, Ingolstadt, Germany. ACM, New York, NY, USA, 6 pages. https://doi.org/10.1145/3473856.3474020

Permission to make digital or hard copies of all or part of this work for personal or classroom use is granted without fee provided that copies are not made or distributed for profit or commercial advantage and that copies bear this notice and the full citation on the first page. Copyrights for components of this work owned by others than ACM must be honored. Abstracting with credit is permitted. To copy otherwise, or republish, to post on servers or to redistribute to lists, requires prior specific permission and/or a fee. Request permissions from permissions@acm.org.

MuC '21, September 5-8, 2021, Ingolstadt, Germany

(C) 2021 Association for Computing Machinery.

ACM ISBN 978-1-4503-8645-6/21/09 ..\$15.00

https://doi.org/10.1145/3473856.3474020

\section{INTRODUCTION}

One cornerstone of a just society are equal job opportunities. Depending on the type of job, this requires accessibility in many different areas: physical access, way finding and many more. In the context of office work, business meetings are particularly critical. One special case are brainstorming meetings; a scenario selected for this work due to the manifold nature of social interaction, including non-verbal communication, and various sources information: Digital ones, like digital whiteboards, PowerPoint presentations, as well as analog ones, like flipcharts. Digital accessibility has made significant strides towards universal access, positively affecting many target groups including blind and visually impaired people. In particular, web accessibility has a solid and well-established basis with the WCAG guidelines, currently in revision 2.1 [27]. Software accessibility, found on desktop operating systems and mobile devices like smartphones and tablets, has also come a long way in recent years. Most providers of graphical user interface frameworks have considered accessibility to some extent. This is also a positive development for the accessibility of business meetings. Tool support in this area has shifted over the course of the last years from analog means, like flipcharts, to digital ones including mind map tools, group decision making tools or brainstorming tools. This creates the possibility to make this type of content fully accessible to a broader range of participants, including blind and visually impaired people (BVIP) in the future. However, one essential part is still an enormous barrier for this group of people; non-verbal and spatial aspects in this scenarios. During conversations, people heavily make use of gestures, postures and facial expressions to give necessary context to other participants. Meeting rooms are usually equipped with whiteboards and screens, and people refer to these objects or even pieces of information on them with deictic gestures like pointing gestures. For visually impaired and especially for blind people, this information is not accessible, excluding them from a major part of the conversation and that makes it difficult to follow. Additionally, information is generated in parallel on multiple levels, resulting in a multi-dimensional information space. In contrast, common techniques of BVIPs to access digital information are all linear, for example, audio output via screenreaders or braille displays. This paper presents a full user interface approach and a functional prototype named MAPVI brainstorming tool as part of the MAPVI project [9], which tackles these shortcomings and allows BVIPs to access spatial, visual and non-verbal information of business meetings. 


\section{RELATED WORK}

Business meetings are an essential activity of the professional work life. There are numerous tools to support, structure and document lively discussions, ranging from analog whiteboards, sketching tables and flipcharts to more modern variations using screens with keyboards, mice, digital pens or touch screens. While these tools can already generate a benefit for BVIP as some of them can save information in a textual format, many of them store visual information, like handwriting, drawings, illustrations or the spatial arrangement of items on a whiteboard, inaccessibly to this group of people. Researchers developed over the years approaches to solve or at least mitigate this issue. Earlier ones usually used mice and keyboards for user interaction, some even involved optically tracked pens[6] and PDAs[18] to transfer information to the system, which later on was displayed on a shared electronic whiteboard. A more recent approach facilitates a back-projected interactive table and interactive vertical screens[11]. A more comprehensive overview of augment work environments can be found in $[4,16]$. However, visual artifacts of meetings rooms are only one domain that is mostly inaccessible to BVIPs. Spatial information about artifacts during business meetings actually offer a much greater challenge. Prior research efforts were concerned with world exploration techniques for BVIPs, using different approaches $[1,3,7,10,30]$. Alongside explicit research for BVIPs, multiple user interface approaches focusing on exploring and manipulating information in AR have been developed in recent years like ARPen[28] using ARCore[8], or T(ether)[17]. While not having this target group in mind, these approaches still seem interesting and parts can be adapted for this group of people. One important issue to note here is that it is of upmost importance to avoid overloading the auditory channel of BVIPs. User interface concepts that heavily rely on this channel mostly come with the disadvantage that users have to decide to either pay attention to the device and its user interface, or to participate in a conversation. While braille displays are an established tool within the community of BVIP that can circumvent this issue, these devices are best suited for linear text. There are however variations of braille displays, like the HyperBraille pad[23], that offer a two-dimensional array of braille characters. Additionally, it was extended via audio notifications and by adapting tangible interaction concepts[24]. While this might offer a usable user interface for BVIPs in certain situations to convey spatial aspects of information domains it still comes with some disadvantages like a high price and a relatively low resolution. Moreover, it does not address the issue at hand, the accessibility of business meetings, as in addition to spatial information, non-verbal communication also plays a central role in this context. Experts in this field believe that up to $55 \%$ of all human communication is non-verbal[19]. The interpretation of body language allows people to get an insight of a peer's emotions and attitude, which in return leads to an increased effectiveness in communication[12] Tremendous amounts of research labor has been put into this topic to sense all kinds of non-verbal communication, including body postures, body language, hand gestures, pointing gestures, facial expressions and eye gestures[2, 5, 20-22, 26]. While all of this, visual information, spatial information and non-verbal information, has the potential to drastically improve the accessibility of business meetings to BVIPs, no comprehensive user interface approach has been found that covers all of these domains of information in an easy-to-use fashion.

\section{THE SCENARIO}

This paper describes a novel and complete user interface approach, which aims at providing access for BVIPs to domains of information of business meetings previously inaccessible to them, which is actively hampering them in their professional life. Business meetings do not look the same, depending on the context, the company and the branch. For this project, a concrete scenario was selected, which is considered to offer a broad variety of types of information; namely brainstorming meetings: In this scenario, multiple participants are present in a meeting room, and multiple people are participating remotely. One or more of these participants, locally or remotely, can be BVIPs. In the meeting room, there are multiple screens mounted to walls. They can have touch functionality. Information is shared on these screens as graph. This graph consists of notes containing a title and text and can be arbitrarily placed in a 2D space. These notes can be linked with each other and can be grouped as well. People present in the meeting room, primarily sit around a table, but they can walk to a screen to emphasize on an argument they are making or moderate a discussion, changing their position in the room. They can use gestures and point at screens, and more specifically on items displayed on the screens. Analyzing this scenario, there is a multitude of types of information, which needs to be considered. Most notable is the existence of two different layers of spatial information:

- One information space is the actual meeting room: participants have names, a position, perform gestures and other types of non-verbal communication. Screens are mounted to walls at a certain position and participants stand close to these screens and are pointing at them.

- The second information space are whiteboards. Notes are located at a certain position in this 2D space, are placed close to each other, above each other or besides each other. These spatial arrangements all bear a certain meaning, depending on the context.

\section{METHODOLOGY}

User acceptance is one of the main success criteria of every user interface approach. Hence, we involved users of the target group from the beginning of the project, starting with questionnaires (3 legally blind and 6 visually impaired)[13]. Additionally, the research unit had and has direct access to two legally blind people during the prototyping process. According to these efforts, NVC and spatial aspects of business meetings are crucial for a full participation. These findings were in line with the results of a previous project[15], which was also concerned with the accessibility of brainstorming meetings, where a lack of accessibility in non-verbal and spatial information was identified at the time. Previous research, which again involved blind and visually impaired people, showed promising results for parts of the whole user interface concept. A project[25], similar to the smartphone integration presented here, was developed and tested at our research unit with blind people to allow them to haptically explore and identify virtual 3D shapes with a smartphone. Another project[14], which was concerned with 

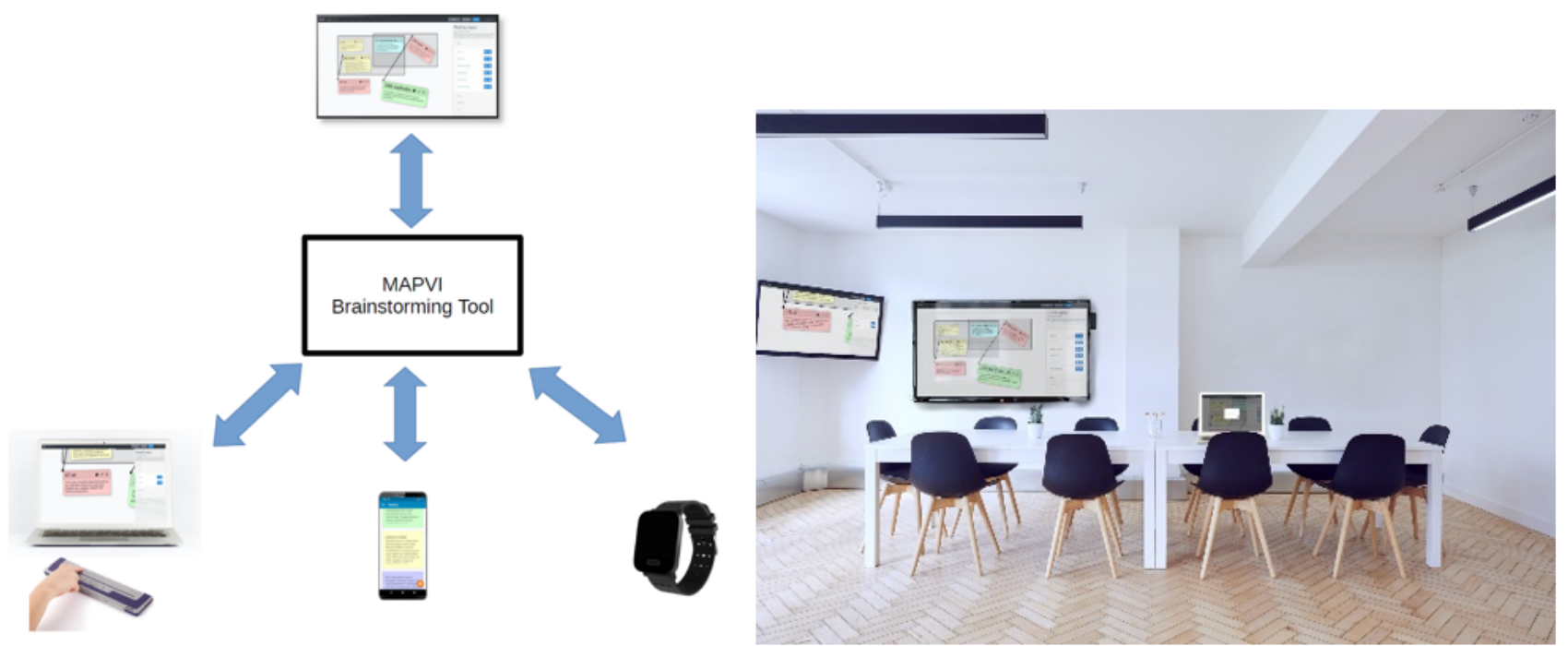

Figure 1: Left side: Concept of devices connecting to MAPVI brainstorming tool. Right side: Meeting room with screens and notebook running the MAPVI web frontend

mobility assistance of BVIPs, used smartphones in a similar fashion than the smartwatch is integrated in this work. There users could explore the environment by pointing the smartphone in the direction of potentially interesting locations ahead or search for points of interest. This was also tested with multiple blind and visually impaired students and was found to be very promising. As a next step and due to the lifting of COVID restriction, user testing and involvement on a broader scale with the existing prototype is possible and will be undertaken the next months.

\section{OVERVIEW OF THE CONCEPT}

While screenreaders, often used with linear braille displays, are established assistive technology for BVIPs to retrieve digital information. This type of user interaction is by nature most effective for one-dimensional information. Even though there are devices like 2D braille displays, they have barely been adopted yet. This paper presents an alternative approach using affordable off-the-shelf hardware for this user interaction approach to provide access to both layers of spatial, non-verbal and visual information. This approach has already been implemented as fully functional prototype called MAPVI brainstorming tool (see Figure 1). Information can be retrieved, display and manipulated in manifold ways. A web interface provides easy access to all information on a broad variety of hardware, like desktop computers, notebooks, tablets and smartphones. While this interface already allows for a very flexible and broadly accessible approach to access all kinds of information related to a meeting, spatial attributes can still not be communicated to BVIPs in a user-friendly fashion. This is why smartphones and smartwatch are utilized for this user interface concept in addition. A smartphone app lets the user explore and manipulate whiteboard information. A smartwatch app provides users with an easy-to-use interface to retrieve information of real world objects and persons. A server infrastructure, called MAPVI brainstorming tool, centrally stores the data of all meetings, and of every information related to each meeting (see Figure 1). All other client devices connect to this infrastructure using a REST service to retrieve and manipulate data and a web socket service for real-time events.

\section{WEB FRONTEND}

The web frontend, which is one way to access meeting information, connects to the server. Desktop computers and notebooks can display the virtual whiteboard of a meeting. Multiple views on the same whiteboards can be used to show different portions at different zoom levels across multiple screens and devices. The web interface is accessible as well as responsive (see Figure 2). This means BVIPs can use screenreaders and braille displays to access this information, but also participants with smartphones or tablets can use the same web interface to access the same information in a layout optimized for their screen size. The web interface is accessible to sighted keyboard users as well as BVIP using smartphones or tablets as well and works with touch screens.

\section{SMARTPHONE}

Since traditional user interface approaches for BVIPs, like the web interface, excel at displaying textual, one-dimensional content, but are struggling with spatial, two-dimensional information, a new approach facilitating modern off-the-shelf smartphones has been created. A smartphone app for Android has been developed in Unity utilizing Google's augmented reality framework ARCore[8] to grand access to spatial aspects of whiteboard information as well. BVIPs can use it to explore notes on the whiteboard haptically, which has shown promising results in prior research[25]. Depending on the position on the whiteboard, virtual 3D objects are created as their surrogates. BVIPs can move the smartphone, they are holding in the hand, on a virtual plane erect in front of them. If they come close to a note, they get a subtle haptic feedback via the vibration motors of the phone and if they touch the surrogate of the note, 


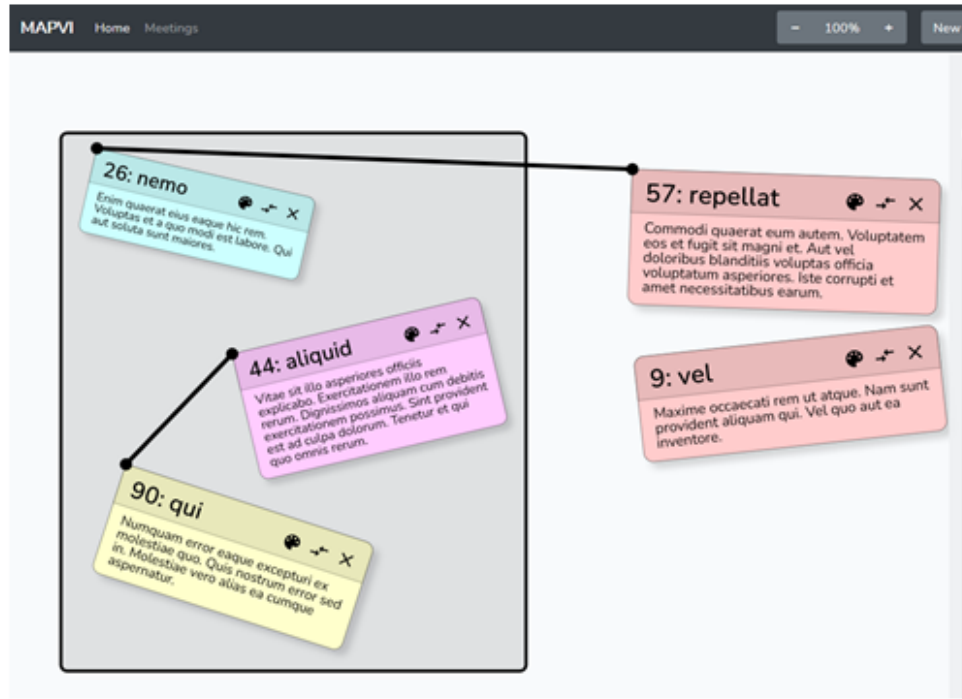

Hew Growe co Busk

Meeting: quod

Figure 2: Left side: the web fronted in desktop mode. Right side: web frontend in smartphone mode.

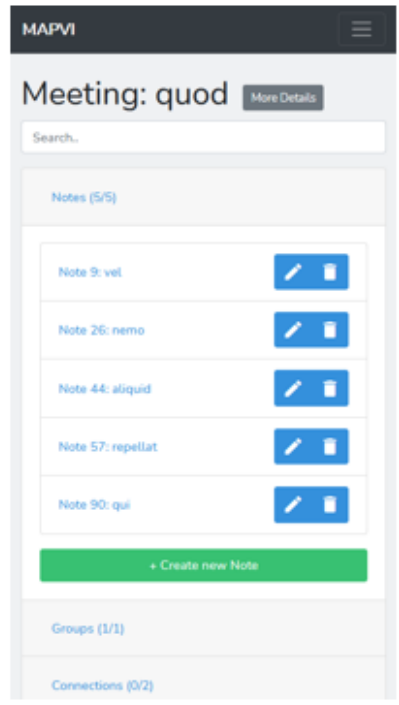

they get a stronger haptic feedback. This way, they can explore the whole virtual whiteboard haptically and directly experience which notes are next to each other, which notes are above each other and what the relative distance between multiple notes is. However, exploration is only one part of this approach. Pressing the hardware buttons on the side of the phone in a grip-like fashion they can also retrieve information about a note and even manipulate it. By clicking the volume-up key they can retrieve additional information of the note like the title and the body text, via cloud-based text to speech. By holding the same key they can naturally move and rotate a note by moving and rotating the phone. The volume-down key allows BVIPs to highlight elements on the whiteboard for all other participants of the meeting. Clicking the button, visually and logically for other blind participants, highlights the current note the person is virtually touching with the smartphone. Holding the same button displays a visual cursor appears on the whiteboard for all other participants in case they want to draw the attention to whole area rather than one element.

\section{SMARTWATCH}

As the AR-based smartphone approach only covers one level of spatial information, another category of devices was integrated as well, namely smartwatches. Another application developed for Android Wear[29] allows compatible smartwatches to be used as user interface devices in combination with the web interface to retrieve additional information in the meeting room, explore the environment and search for real-world objects. This requires real-time indoor localization, which is out of the scope of this work, since it focuses on the user interface concept. However, the REST-interface allows real-time localization data to be feed into the system easily, and basically all approaches can be integrated, as long as they rely on a geographic coordinate system. For prototyping and evaluation, data is feed into the system manually for now. To retrieve this information, the BVIP is using a notebook, running a screenreader with braille display and/or bone conductive headphones and he or she wears a smartwatch. The user can perform pointing and wrist flick gestures towards points of interest. This can be persons or equipment of the meeting room holding information, like screens or digital whiteboards. The web interface can be switched to an exploration mode to show information related to objects the person is pointing at. This gives BVIP access to spatial information usually reserved to sighted participants only. Blind and visually impaired participants can answer following questions with it, without interrupting the actual conversation of the meeting:

- "People have changed seats after the break. Who is the person sitting across the table now?"

- "To which screen is the person pointing, who currently is talking?"

- "Where is the person sitting I want to direct my next question at?"

In particular, three modes of operation are currently implemented:

- Exploration mode: The user, with the smartwatch strapped on, can move the hand in any direction. If a point of interest is ahead, the smartwatch starts vibrating and the web interface, the same user is connected to, shows a short summary of what is ahead.

- Retrieval mode: If an object or person is ahead, the user can perform a wrist flick, which the smartwatch recognizes and the web interface shows additional information of the point of interest.

- Search mode: this mode can be activated in the web interface for certain elements, namely participants of a meeting and real-world objects like screens. E.g. If the user activates the mode for a participant, the smartwatch can be used to haptically find the orientation of this person. The closer the user is pointing in the right direction the strong the vibration gets. 

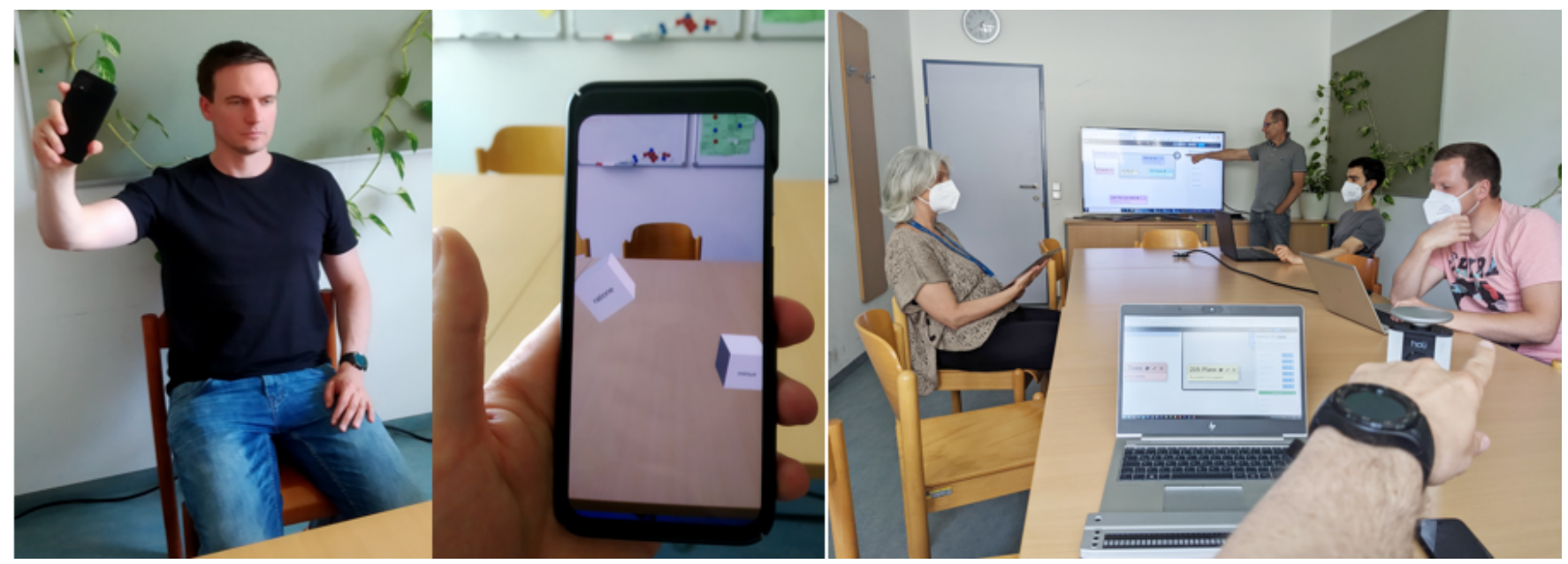

Figure 3: Left image: person using the smartphone to explore the virtual whiteboard haptically, center image: note surrogates displayed in AR smartphone app, Right image: person using smartwatch to point at physical objects or person to retrieve additional information via the web frontend

\section{CONCLUSION AND FUTURE WORK}

In this paper, we presented a complete user interface concept and a functional prototype based on it, which aims at making business meetings more accessible to blind and visually impaired people (BVIP). For this approach, we use affordable off-the-shelf hardware, with smartphones and smartwatches, to create an intuitive and accessible user interface for this group of people, which allows them to not only hear other participants, but also get access to normally inaccessible information including spatial aspects and non-verbal information of a business meeting. While blind people were involved from the beginning in the development of the concept and the prototype, it has not been tested at a broader scale. Therefore, the next major step are evaluations to study the benefit for BVIPs as well as highlight areas where the concept or the prototype can be improved.

\section{ACKNOWLEDGMENTS}

This project (MAPVI) including this publication was funded by the Austrian Science Fund (FWF): I 3741-N31.

\section{REFERENCES}

[1] Richard A. Bolt. 1980. "Put-that-there". In Proceedings of the 7th annual conference on Computer graphics and interactive techniques - SIGGRAPH '80, James J. Thomas, Robert A. Ellis, and Harvey Z. Kriloff (Eds.). ACM Press, New York, New York, USA, 262-270. https://doi.org/10.1145/800250.807503

[2] S. M. Bopalkar, P. Talwai, and B. H. Parmar. 2011. Body parts detection in gesture recognition using color information. In Proceedings of the International Conference \& Workshop on Emerging Trends in Technology, B. K. Mishra (Ed.). ACM, New York, NY, 149. https://doi.org/10.1145/1980022.1980054

[3] Michael Brock and Per Ola Kristensson. 09082013. Supporting blind navigation using depth sensing and sonification. In Proceedings of the 2013 ACM conference on Pervasive and ubiquitous computing adjunct publication, Friedemann Mattern, Silvia Santini, John F. Canny, Marc Langheinrich, and Jun Rekimoto (Eds.). ACM, New York, NY, USA, 255-258. https://doi.org/10.1145/2494091.2494173

[4] Sebastian Büttner, Henrik Mucha, Markus Funk, Thomas Kosch, Mario Aehnelt, Sebastian Robert, and Carsten Röcker. 06212017. The Design Space of Augmented and Virtual Reality Applications for Assistive Environments in Manufacturing In Proceedings of the 10th International Conference on PErvasive Technologies
Related to Assistive Environments. ACM, New York, NY, USA, 433-440. https: //doi.org/10.1145/3056540.3076193

[5] Hong Cheng, Lu Yang, and Zicheng Liu. 2016. Survey on 3D Hand Gesture Recognition. IEEE Transactions on Circuits and Systems for Video Technology 26, 9 (2016), 1659-1673. https://doi.org/10.1109/TCSVT.2015.2469551

[6] Scott Elrod, Ken Pier, John Tang, Brent Welch, Richard Bruce, Rich Gold, David Goldberg, Frank Halasz, William Janssen, David Lee, Kim McCall, and Elin Pedersen. 1992. Liveboard. In Proceedings of the SIGCHI conference on Human factors in computing systems - CHI '92, Penny Bauersfeld, John Bennett, and Gene Lynch (Eds.). ACM Press, New York, New York, USA, 599-607. https://doi.org/10.1145/142750.143052

[7] Michele Geronazzo, Alberto Bedin, Luca Brayda, Claudio Campus, and Federico Avanzini. 2016. Interactive spatial sonification for non-visual exploration of virtual maps. International fournal of Human-Computer Studies 85 (2016), 4-15. https://doi.org/10.1016/j.ijhcs.2015.08.004

[8] Google Developers. 09.09.2020. Build new augmented reality experiences that seamlessly blend the digital and physical worlds. https://developers.google.com/ ar

[9] Sebastian Gunther, Reinhard Koutny, Naina Dhingra, Markus Funk, Christian Hirt, Klaus Miesenberger, Max Mühlhäuser, and Andreas Kunz. 2019. MAPVI. In Proceedings of the PETRA'19, 12th ACM International Conference on Pervasive Technologies related to Assistive Environments, Rhodes, Greece June 05-07, 2019, Fillia Makedon (Ed.). ACM = Association for Computing Machinery, New York, 343-352. https://doi.org/10.1145/3316782.3322747

[10] Anhong Guo, Xiang 'Anthony' Chen, Haoran Qi, Samuel White, Suman Ghosh, Chieko Asakawa, and Jeffrey P. Bigham. 10162016. VizLens. In Proceedings of the 29th Annual Symposium on User Interface Software and Technology, Jun Rekimoto, Takeo Igarashi, Jacob O. Wobbrock, and Daniel Avrahami (Eds.). ACM, New York, NY, USA, 651-664. https://doi.org/10.1145/2984511.2984518

[11] Hans-Christian Jetter, Harald Reiterer, and Florian Geyer. 2014. Blended Interaction: understanding natural human-computer interaction in post-WIMP interactive spaces. Personal and Ubiquitous Computing 18, 5 (2014), 1139-1158. https://doi.org/10.1007/s00779-013-0725-4

[12] Markus Koppensteiner, Pia Stephan, and Johannes Paul Michael Jäschke. 2016. Moving speeches: Dominance, trustworthiness and competence in body motion. Personality and Individual Differences 94 (2016), 101-106. https://doi.org/10.1016/ j.paid.2016.01.013

[13] Reinhard Koutny, Sebastian Günther, Naina Dhingra, Andreas Kunz, Klaus Miesenberger, and Max Mühlhäuser. [n.d.]. Accessible Multimodal Tool Support for Brainstorming Meetings. https://doi.org/10.3929/ETHZ-B-000440125

[14] Reinhard Koutny and Klaus Miesenberger. 2015. Virtual mobility trainer for visually impaired people. Technology and Disability 26, 4 (2015), 211-219. https: //doi.org/10.3233/TAD-140420

[15] Andreas Kunz, Klaus Miesenberger, Max Mühlhäuser, Ali Alavi, Stephan Pölzer, Daniel Pöll, Peter Heumader, and Dirk Schnelle-Walka. 2014. Accessibility of Brainstorming Sessions for Blind People. In Computers Helping People with Special Needs, David Hutchison, Takeo Kanade, Josef Kittler, Petr Penaz, and Wolfgang Zagler (Eds.). Lecture Notes in Computer Science / Information Systems 
and Applications, Incl. Internet/Web, and HCI, Vol. 8547. Springer International Publishing, Cham, 237-244. https://doi.org/10.1007/978-3-319-08596-8\{_\}38

[16] Saadi Lahlou (Ed.). 2009. Designing user friendly augmented work environments From meeting rooms to digital collaborative spaces. Springer, London and New York.

[17] David Lakatos, Matthew Blackshaw, Alex Olwal, Zachary Barryte, Ken Perlin, and Hiroshi Ishii. 2014. T(ether). In Proceedings of the 2014 Symposium on Spatial User Interaction, October 4 - 5, 2014, Honolulu, Hawaii, USA, Andy Wilson (Ed.) ACM, New York, NY, 90-93. https://doi.org/10.1145/2659766.2659785

[18] Carsten Magerkurth and Thorsten Prante. 2001. „Metaplan“ für die Westentasche: Mobile Computerunterstützung für Kreativitätssitzungen. In Mensch \& Computer 2001, Wolf-Rüdiger Gawron, Günter Riedewald, Eckard Jaus, Roland Dürre, Horst Oberquelle, Reinhard Oppermann, and Jürgen Krause (Eds.). Berichte des German Chapter of the ACM, Vol. 55. Vieweg+Teubner Verlag, Wiesbaden, 163-171. https://doi.org/10.1007/978-3-322-80108-1\{_\}18

[19] Albert Mehrabian. 2007. Nonverbal Communication (1st ed. ed.). Taylor and Francis, Somerset. https://ebookcentral.proquest.com/lib/gbv/detail.action?docID= 4930823

[20] Akilesh Rajavenkatanarayanan, Ashwin Ramesh Babu, Konstantinos Tsiakas, and Fillia Makedon. 2018. Monitoring task engagement using facial expressions and body postures. In Proceedings of the 3rd International Workshop on Interactive and Spatial Computing - IWISC '18, Prabhakaran Balakrishnan and Ryan P. McMahan (Eds.). ACM Press, New York, New York, USA, 103-108. https://doi.org/10.1145/ 3191801.3191816

[21] Siddharth S. Rautaray and Anupam Agrawal. 2015. Vision based hand gesture recognition for human computer interaction: a survey. Artificial Intelligence Review 43, 1 (2015), 1-54. https://doi.org/10.1007/s10462-012-9356-9

[22] S. Qiu, J. Hu, and G. Rauterberg. 2015. Nonverbal Signals for Face-to-Face Communication between the Blind and the Sighted. undefined (2015) https://www.semanticscholar.org/paper/Nonverbal-Signals-for-Face-to-Face-
Communication-Qiu-Hu/9e49a9cd7cf3e610106dd2093590c42bad98e924\#citingpapers

[23] Stephan Pölzer and K. Miesenberger. 2014. A Tactile Presentation Method of Mind Maps in Co-located Meetings. undefined (2014). https://www. semanticscholar.org/paper/A-Tactile-Presentation-Method-of-Mind-Maps-inP\%C3\%B6lzer-Miesenberger/60985828e99a0890c864fc134babd3d4beecd80c

[24] U. Kannengiesser and Stefan Oppl. 2015. Business Processes to Touch: Engaging Domain Experts in Process Modelling. undefined (2015). https:// www.semanticscholar.org/paper/Business-Processes-to-Touch\%3A-EngagingDomain-in-Kannengiesser-Oppl/db888b0266ff74eb850ebb4639f182b6c60da47f

[25] Verma Aashish, Miesenberger Klaus, Pertl Gregor, Reithmayr Kerstin. [n.d.]. Use of Mobile Devices for Haptic Interaction for classifying 3D Geometrical Objects. In Proceedings of the 4th International Workshop on "Digitization and E-Inclusion in Mathematics and Science 2021".

[26] Alessandro Vinciarelli. 2017. Body language without a body: nonverbal communication in technology mediated settings. In ISIAA'17, Thierry Chaminade (Ed.). The Association for Computing Machinery, Inc, New York, NY, 2-3. https://doi.org/10.1145/3139491.3139510

[27] W3C. 05.06.2018. Web Content Accessibility Guidelines (WCAG) 2.1. https: //www.w3.org/TR/WCAG21/

[28] Philipp Wacker, Oliver Nowak, Simon Voelker, and Jan Borchers. 2019. ARPen. In CHI 2019, Stephen Brewster, Geraldine Fitzpatrick, Anna Cox, Vassilis Kostakos, and Anna L. Cox (Eds.). The Association for Computing Machinery, New York, New York, 1-12. https://doi.org/10.1145/3290605.3300849

[29] Wear OS. 11.05.2021. Wear OS by Google Smartwatches. https://wearos.google. com/\#hands-free-help

[30] S. Willis and S. Helal. 18-21 Oct. 2005. RFID Information Grid for Blind Navigation and Wayfinding. In Ninth IEEE International Symposium on Wearable Computers (ISWC'05). IEEE, 34-37. https://doi.org/10.1109/ISWC.2005.46 